\title{
Bond-bias simulation of phase equilibria for strongly associating fluids
}

\author{
D. M. Tsangaris and J. J. de Pablo \\ Department of Chemical Engineering, University of Wisconsin-Madison, Madison, Wisconsin 53706
}

(Received 7 July 1993; accepted 28 March 1994)

\begin{abstract}
In this work a novel Monte Carlo method is developed to simulate the equilibrium thermodynamic properties of strongly associating fluids. The highly anisotropic nature of intermolecular interactions in these fluids makes conventional simulation techniques of little use. By introducing biased sampling techniques we are able to explore configuration space efficiently, thereby obtaining reliable estimates for the thermodynamic properties, including phase equilibria, of model systems. The results of our simulations are used to assess the accuracy and validity of various theories for associating fluids.
\end{abstract}

\section{INTRODUCTION}

The modeling of associating fluids has been an active area of research for several decades. In this period of time, attention has gradually shifted from the so-called chemical theories, where molecular association is treated as a chemical reaction, to molecular models where association arises naturally from strong attractive intermolecular forces. ${ }^{1}$ Molecular models, consisting of simple shapes with sites that interact with each other through short ranged but strongly orientational dependent intermolecular forces, have in recent years been shown to capture the main characteristics of associating fluids.

However, while much progress has been made with analytical theories for such systems, attempts to simulate them (and verify the theories) have been scarce. The lack of simulation work in this area can be attributed to the difficulties associated with sampling the configurational space of associating fluids, particularly for high association strengths. In this work we propose a new simulation method that greatly reduces such difficulties.

The advent and success of such models is largely due to Wertheim, who recognized that steric incompatibilities in associating fluids lead to the cancellation of a certain class of graphs in the cluster expansion for the fugacity. ${ }^{2,3}$ When expressed as a function of the densitics of monomers and of bonded species, Wertheim's expansion is able to describe accurately the association phenomena that occur in such fluids. By combining Wertheim's theory of association with other theories of simple systems, it is possible to predict the thermodynamic properties of model associating liquids and gases.

Early molecular models that relied on hard-sphere reference fluids were not able to predict "true" vapor-liquid equilibria unless there was significant percolation from the association part of the intermolecular potential. ${ }^{4}$ It appears that phase transitions can only be obtained when dispersive forces are taken into account explicitly. ${ }^{5}$ One such model, which uses the Lennard-Jones fluid as a reference, was proposed by Chapman ${ }^{6}$ and subsequently studied by Johnson and Gubbins. ${ }^{7}$ These authors, however, restricted their study to fluids with weak to moderate associating forces.

In this work we show that the forces required to describe the thermodynamic properties (including phase equilibria) of common associating fluids such as carboxylic acids are often much stronger than those investigated by Johnson and Gubbins. We use a model similar to that of these authors but we study much higher association strengths. To assess the accuracy of the theory for prediction of the saturation properties of these fluids we perform molecular simulations in the Gibbs ensemble. Note that conventional Gibbs-ensemble simulations can only yield reliable results for weakly associating fluids. For strongly associating fluids, the formation or destruction of bonds becomes a slow process for which conventional sampling techniques are of little use. Here, we propose a novel simulation method that overcomes the aforementioned sampling problems and facilitates the simulation of strongly associating fluids.

The new method, which we call bond-bias Monte Carlo (BBMC), biases trial insertion moves in the direction of bond formation, thereby crossing over the large energy barriers that separate the formation and destruction of chemical bonds. The crossing of these barriers is achieved by inserting monomers into selected areas of the simulation box.

The organization of this manuscript is as follows. Following this Introduction, the second section begins with a description of the model and the thermodynamic perturbation theory that we use to calculate its thermodynamic properties. Next, after providing a brief overview of simulations in the Gibbs ensemble, we present our BBMC method and we derive the corresponding governing equations. The fourth section begins by comparing the results of such a method to those of conventional Gibbs ensemble simulations for weakly associating fluids. We subsequently present results for strong association strengths, and we compare them to the predictions of the theory. To substantiate our argument that strong association strengths are required to model the thermodynamic properties of real fluids, we also use the theory to describe experimental data for acetic acid. Finally, a third section presents some concluding remarks concerning both the validity of the theory and the extent of applicability of the BBMC method presented in this work.

\section{THEORY}

\section{A. Molecular model}

Vapor-liquid equilibria for associating pure fluids are studied using a molecular model consisting of a simple 


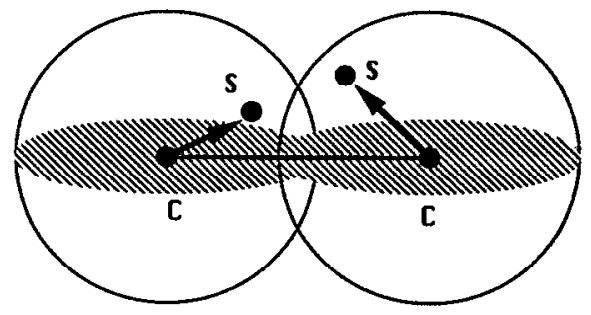

FIG. 1. Molecular model.

sphere with an off-center attraction site. Molecules interact with each other through a combination of Lennard-Jones (12-6) intermolecular interactions and a square-well attraction.

More specifically, two particles $i$ and $j$ interact through a potential energy function of the form

$$
\Phi_{i j}\left(r_{i j}\right)=\phi_{\mathrm{cc}}\left(r_{i j}\right)+\phi_{\mathrm{ss}}\left(r_{i j}, \omega_{i}, \omega_{j}\right),
$$

where $r_{i j}$ denotes the center-to-center distance between the particles. The first term in Eq. (1) is given by

$$
\phi_{\mathrm{cc}}(r)=4 \epsilon\left[\left(\frac{\sigma}{r}\right)^{12}-\left(\frac{\sigma}{r}\right)^{6}\right] \text {. }
$$

The second term in Eq. (1) corresponds to attractive site-site interactions and is given by

$$
\phi_{\mathrm{ss}}=\left\{\begin{array}{c}
-\epsilon_{\mathrm{sw}} \quad \text { if } r_{0}<r<r_{c} \\
\theta_{i}<\theta_{c} \text { and } \theta_{j}<\theta_{c} . \\
0 \quad \text { otherwise }
\end{array}\right.
$$

Attraction sites are located at a distance $l$ from the center of the sphere. As Eq. (3) indicates, attractions (with well depth $\epsilon_{\mathrm{sw}}$ ) are effective only when molecules are separated by a distance smaller than $r_{c}$ and when the angle between the center-center and the center-site vectors is less than $\theta_{c}$ (see Fig. 1). An additional condition, which in not apparent from Eq. (3), requires the center-site vectors to "face" one another as shown in Fig. 1. There are no intramolecular or intermolecular interactions between a center and a site. In addition to $\sigma$ and $\epsilon$, the Lennard-Jones potential parameters, $r_{c}, \theta_{c}$, and $\epsilon_{\mathrm{ss}}$ are also parameters of the model. The total internal energy of the system is given by

$$
U=\frac{1}{2} \sum_{i=1}^{i=N} \sum_{j=1}^{j=N} \Phi_{i j},
$$

where $\Phi_{i j}$ is given by Eq. (1).

\section{B. Thermodynamic perturbation theory}

The Helmholtz free energy and the pair correlation function of the reference system provide the basis for the thermodynamic perturbation theory (TPT) discussed here. This free energy is expressed as a sum of two terms. The first term takes into account the properties of the reference system and the second term takes into account corrections due to association. The theory requires two densities; the total-number density of the system and the density of the molecules that are not bonded (monomers). Corrections to the Helmholtz free energy are then expressed as a function of the pair correlation function of the reference fluid and the above densities. All other thermodynamic properties can be obtained from the Helmholtz free energy using thermodynamic manipulations. Note, however, that this theory does not provide any structural information (such as pair correlation functions) about the system.

According to the theory, for a system with multiple association sites, the Helmholtz residual energy (with respect to the ideal gas) is given by the following expression:

$$
\frac{A^{R}}{N k T}=\frac{A^{R, \text { ref }}}{N k T}+\frac{A^{\text {Assoc }}}{N k T},
$$

where

$$
\frac{A^{\mathrm{Assoc}}}{N k T}=\sum_{A}\left[\ln x_{A}-\frac{x_{A}}{2}\right]+\frac{M}{2}
$$

and where $M$ is the number of associating sites per molecule, $x_{A}$ is the fraction of molecules not bonded at site $A$, and $A^{R \text {,ref }}$ is the residual Helmholtz energy of the reference system. The summation is over all available association sites on the molecule.

For the $x_{A}$ we have

$$
x_{A}=\left[1+\sum_{B} \rho x_{B} \delta_{A B}\right]^{-1},
$$

where $\rho$ is the number density of the system and where the summation is over all available association sites except $A$. For $\delta_{A B}$ we use

$$
\delta_{A B}=\int g^{\mathrm{ref}}(12) f(12) d(12),
$$

where

$$
f_{A B}=\exp \left[-\phi_{A B}(12) / k T\right]-1
$$

and where $g^{\text {ref }}(12)$ is the pair correlation function of the reference fluid. The integration $d(12)$ is to be performed over all possible orientations and separations (unweighted average) between a pair of molecules 1 and 2 .

For the "single-association-site" model shown in Fig. 1, $\delta_{A B}$ simplifies to

$$
\delta \equiv \delta_{A B}=\int g^{\mathrm{ref}}(12)\left[\exp \left(-\phi_{\mathrm{ss}} / k T\right)-1\right] d(12)
$$

which, after carring out the integration, reduces to

$$
\delta=\pi\left(1-\cos \theta_{c}\right) F \int_{0}^{r_{c}} g^{\mathrm{ref}} r^{2} d r
$$

with $F=\exp \left(-\epsilon_{\mathrm{sw}} / k T\right)-1$.

For a single site model, the mole fraction of molecules not bonded at site $A$ (denoted by $x$ hereafter), is actually equivalent to the mole fraction of monomer and, according to Eq. (7), it is given by

$$
x=[1+x \rho \delta]^{-1} \text {. }
$$


The above equation is quadratic with respect to $x$ and simplifies further to

$$
\begin{aligned}
x & =\frac{\sqrt{1+4 \rho \delta}-1}{2 \rho \delta}, \\
& =\frac{2}{\sqrt{1+4 \rho \delta}+1} .
\end{aligned}
$$

Finally, from Eq. (6) we obtain

$$
\frac{A^{\text {Assoc }}}{N k T}=\ln x-\frac{x}{2}+\frac{1}{2}
$$

or after substitution

$$
\frac{A^{\text {Assoc }}}{N k T}=\ln \frac{\sqrt{1+4 \rho \delta}-1}{2}-\frac{\sqrt{1+4 \rho \delta}-1}{2}+\frac{1}{2} .
$$

Equations (11), (13), and (14), along with an expression for the pair distribution function of the Lennard-Jones fluid, are sufficient to describe the contributions of attractive forces to the thermodynamic properties of the system. In this work, the pair distribution function of the Lennard-Jones fluid was obtained using the WCA approximation ${ }^{8}$ and a semiempirical formula proposed by Johnson and Gubbins. ${ }^{7}$ The latter formulation leads to more accurate pair distribution functions in the low-density regime. In the high-density regime, both formalisms lead to the same results for $g(r)$. The thermodynamic properties of the Lennard-Jones reference fluid were obtained using the newly reparametrized equation of state of Johnson et al. ${ }^{9}$

\section{Simulation of phase equilibria in the Gibbs ensemble}

Simulations of phase equilibria are generally carried out in the Gibbs ensemble. The simulated system consists of two boxes, one corresponding to each phase; equilibrium is attained by displacing/rotating particles (thermal equilibrium), by exchanging volume (mechanical equilibrium), and by transferring particles between these phases (chemical equilibrium)..$^{10}$ The method can be summarized by the following steps:

(1) Choose a particle at random in every phase and attempt to change its position and its orientation. Calculate the energy change

$$
\Delta \Gamma^{\text {displace }}=U^{\text {new }}-U^{\text {old }}
$$

and accept the new configuration with probability

$$
q=\min \left[1, \exp \left(-\Delta \Gamma^{\text {displace }}\right)\right],
$$

where $\min (a, b)$ denotes the smaller of the two values, $a$ and $b$, and where $U^{\text {new }}$ and $U^{\text {old }}$ are the internal energies of the system before and after the trial move, respectively.

(2) Attempt a volume "transfer" from any randomly selected phase into the other and accept the new configuration with probability

$$
q=\min \left[1, \exp \left(-\beta \Delta \Gamma^{\text {volume }}\right)\right],
$$

where

$$
\begin{aligned}
\Delta \Gamma^{\mathrm{volume}}= & {\left[\Delta U_{(1)}+\Delta U_{(2)}\right]-\frac{N_{(1)}}{\beta} \ln \left[\frac{V_{(1)}^{\text {new }}}{V_{(1)}^{\text {old }}}\right] } \\
& -\frac{N_{(2)}}{\beta} \ln \left[\frac{V_{(2)}^{\text {new }}}{V_{(2)}^{\text {old }}}\right]
\end{aligned}
$$

where $U_{(i)}$ and $V_{(i)}$ are the configurational energy and the volume of phase $i$.

(3) Attempt a particle transfer from a randomly selected phase (e.g., phase 1) into the other phase (phase 2) and accept the transfer with probability

$$
q=\min \left[1, \exp \left(-\beta \Delta \Gamma^{\text {transfer }}\right)\right],
$$

where

$$
\begin{aligned}
\Delta \Gamma^{\text {transfer }}= & {\left[\Delta U_{(1)}+\Delta U_{(2)}\right]-\frac{1}{\beta} \ln \frac{V_{(2)}}{V_{(1)}} } \\
& -\frac{1}{\beta} \ln \frac{N_{(1)}}{N_{(2)}+1} .
\end{aligned}
$$

\section{Bond-bias Monte Carlo method (BBMC)}

Let us assume that at some point in time the simulated system is in a state $i$ specified by the positions/orientations of the particles in the simulation box. Let us also assume that the transition probability for going from state $i$ to a new state $j$ is $\pi_{i j}$, and that the probabilities of being at two different states $i$ and $j$ are $p_{i}$ and $p_{j}$, respectively. For this system, microscopic reversibility is obeyed if

$$
p_{i} \pi_{i j}=p_{j} \pi_{j i} \text {. }
$$

In the Metropolis method, the system is moved from a state $i$ into a new state $j$ through a two-step process. ${ }^{11}$ Initially, a move into state $j$ is proposed with probability $T_{i j}$; the proposed move is subsequently accepted with probability $A_{i j}$. In this method, microscopic reversibility requires that

$$
p_{i} T_{i j} A_{i j}=p_{j} T_{j i} A_{j i} \text {. }
$$

There are various ways to satisfy Eq. (23) in a simulation. Metropolis et al. ${ }^{12}$ proposed the so-called "asymmetric solution" where

$$
\begin{aligned}
& q=\frac{p_{j} T_{j i}}{p_{i} T_{i j}}, \\
& A_{i j}=\min (1, q), \\
& A_{j i}=\min (1,1 / q) .
\end{aligned}
$$

It can be shown that Eq. (24) satisfies Eq. (23) by considering the two possibilities for $q$, i.e., $q>1$ and $q \leqslant 1$.

When Boltzmann's statistics are used, the probability density function for an arbitrary statistical mechanical ensemble is of the form

$$
\rho(\mathbf{x}) \propto \exp [-\beta \Gamma(\mathbf{x})],
$$

where $\beta=1 / k T, \Gamma$ is a generalized energy, and $\mathrm{x}$ is a multidimensional vector containing all the positions/orientations of the molecules in the box. For the canonical ensemble, for example, $\Gamma$ is the configurational energy $U(\mathbf{x})$. For state $i$, 
the probability that the system's $N$ molecules are located in a differential hyper-volume $d \mathbf{x}^{N}$ around point $\mathbf{x}$ of configurational space is of the form

$$
p_{i} \propto \exp [-\beta \Gamma(\mathbf{x})] d \mathbf{x}^{N} .
$$

While $p_{i}$ is imposed by statistical mechanical considerations, the transition probabilities $T_{i j}$ can be arbitrarily designed in such a way as to maximize the efficiency of a simulation method. The choice of $T_{i j}$ is independent of the physical properties of a system, but it can have a significant effect on the convergence properties of the method. In the original Mctropolis algorithm, $T_{i j}$ was given by

$$
T_{i j}=\left\{\begin{array}{c}
0 \text { if } j \text { is not in the neighborhood of } i \\
1 / N_{R} \text { if } j \text { is in the neighborhood of } i \\
\text { (with } N_{R} \text { available states) }
\end{array}\right.
$$

The Metropolis method, selects a state $j$, from the $N_{R}$ available positions (i.e., the volume of the simulation box) with equal probability.

The original Metropolis method, as well as other methods based on similar selection schemes, have proven to be successful for simple fluids having continuous and "soft" interactions. For such fluids, small variations in the position/ orientation of a particle do not lead to significant changes of the configurational energy of the system. However, when simulating fluids that interact with short-range and highly orientational-dependent forces, very small variations of the position/orientation of a particle can cause large changes of the configurational energy.

For the fluids discussed here, a slight orientational change can result in the destruction of a "bond," thereby leading to a state of much higher energy which has little chances of occurring [Eq. (26)]. Therefore, the simulated system can be viewed as a collection of energetically favorable states separated by high energy barriers; our proposed method concentrates sampling on these low energy states which are not easily accessible with conventional techniques but which contribute significantly to the partition function of the system.

For the specific case of simulations in the Gibbs ensemble, instead of inserting a particle into a completely random position/orientation, we favor positions/orientations that result in the creation of a dimer; i.e., a new bond is formed between the newly inserted particle and an existing monomer. It is important to point out, however, that we leave room for conventional random insertions. We show next that our preferential sampling technique satisfies microscopic reversibility by construction.

If we partition the set of $j$ states into the two subsets $j_{a}$ and $j_{b}$ (see Fig. 2), where

$$
\begin{aligned}
& j_{a} \cap j_{b}=\varnothing \\
& j_{a} \cup j_{b}=j
\end{aligned}
$$

and we assume that the $j_{a}$ subset has $Q_{a}$ available positions and the $j_{b}$ has $Q_{b}$ available positions, then any member of $j$ has probability $T_{i j}=1 /\left(Q_{a}+Q_{b}\right)$ of being randomly se-

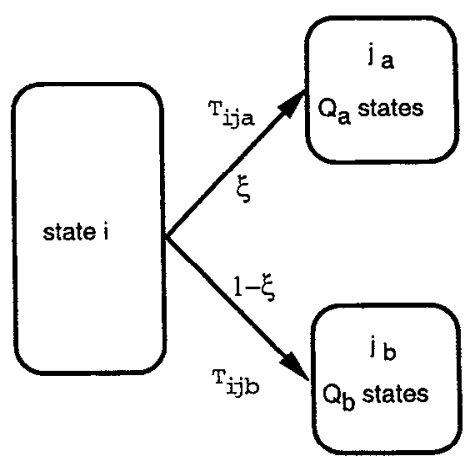

FIG. 2. Partition of $j$ states.

lected. If, on the other hand, we choose any member of $j_{a}$ with probability $\xi$ or any member of $j_{b}$ with probability $1-\xi$, we have

$$
\begin{aligned}
& T_{i j_{a}}=\frac{\xi}{Q_{a}}, \\
& T_{i j_{b}}=\frac{1-\xi}{Q_{b}} .
\end{aligned}
$$

The proposed algorithm satisfies microscopic reversibility if the $i \rightarrow j_{a}$ and $i \rightarrow j_{b}$ transitions are reversible. According to Eq. (23), we must have

$$
\begin{aligned}
& p_{i} T_{i j_{a}} A_{i j_{a}}=p_{j_{a}} T_{j_{a}} i A_{j_{a} i}, \\
& p_{i} T_{i j_{b}} A_{i j_{b}}=p_{j_{b}} T_{j_{b}} A_{j_{b} i} .
\end{aligned}
$$

Along with the modified sampling process we propose the following solution to the above equations; this solution is in complete analogy with the "asymmetric solution" proposed originally by Metropolis:

$$
\begin{aligned}
& q_{a}=\frac{p_{j_{a}} T_{j_{a} i}}{p_{i} T_{i j_{a}}}, \\
& A_{i j_{a}}=\min \left(1, q_{a}\right), \\
& A_{j_{a} i}=\min \left(1,1 / q_{a}\right), \\
& q_{b}=\frac{p_{j_{b}} T_{j_{b}}}{p_{i} T_{i j_{b}}}, \\
& A_{i j_{b}}=\min \left(1, q_{b}\right), \\
& A_{j_{b} i}=\min \left(1,1 / q_{b}\right) .
\end{aligned}
$$

We now show that Eq. (32) are solutions to Eq. (30) for the $i \rightarrow j_{a}$ transition; the other case is symmetric and the proof has been omitted. If $q_{a}<1$, then $A_{i j_{a}}=q_{a}$ and $A_{j_{a} i}=1$ since $1 / q_{a}>1$. Substitution into Eq. (30) gives 


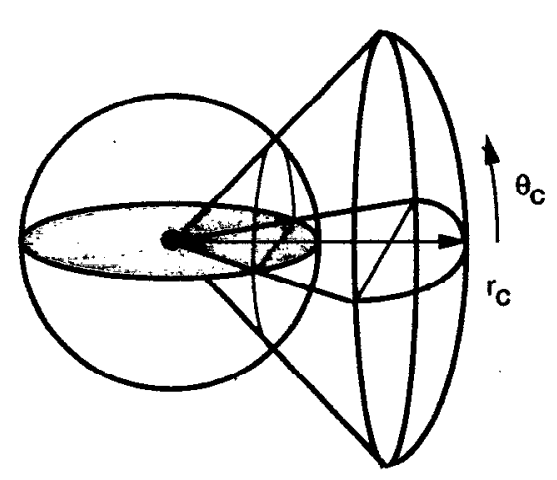

FIG. 3. Effective volume for insertion with dimer formation.

$$
\begin{aligned}
& p_{i} T_{i j_{a}} A_{i j_{a}}=p_{j_{a}} T_{j_{a} i} A_{j_{a} i}, \\
& p_{i} T_{i j_{a}} q_{a}=p_{j_{a}} T_{j_{a} i}, \\
& p_{i} T_{i j_{a}} \frac{p_{j_{a}} T_{j_{a} i}}{p_{i} T_{i j_{a}}}=p_{j_{a}} T_{j_{a} i},
\end{aligned}
$$

which results in the identity

$$
p_{j_{a}} T_{j_{a} i}=p_{j_{a}} T_{j_{a} i} \text {. }
$$

Note that other solutions to Eq. (30) exist but we do not consider them here.

Next, the ideas outlined above are applied to simple Lennard-Jones associating fluids. Note, however, that the method proposed here is not restricted to those fluids and can also be applied to the simulation of fluids interacting with different intermolecular potentials or to fluids with multiple association sites.

According to the intermolecular potential, two particles are considered "bonded" if their center-to-center distance is less than $r_{c}$ and the center-to-site vector is contained within a cone of stereoangle $\theta_{c}$. As shown in Figs. 1 and 3, an insertion close to a monomer ("free" particle) will result in the creation of a new bond and the formation of a dimer if the newly inserted particle is within this cone of radius $r_{c}$ and its center-to-site vector forms an angle with the center-center vector smaller then $\theta_{c}$. We denote this set of "effective" positions by $V_{\text {eff }}$ and the corresponding set of effective orientations of the center-to-site vector by $\Omega_{\text {eff }}$.

For the analysis that follows, we take advantage of the fact that the effective volumes around the insertion points cannot overlap. If there are positions/orientations in the simulation hyper box that belong to the effective area of two (or more) particles, then an insertion in any of these points will result in multiple bonding; the newly inserted particle will be bonded to all the particles that define the areas that overlap. This, however, is prohibited by the construction of the molecular model ${ }^{6}$ and therefore overlaps between these hyper volumes cannot exist. Therefore, the probability of an "effective" insertion in a "cube" of volume $d \mathbf{r}$ and with orientation with "volume" $d \omega$ is

$$
d \mathbf{r} d \boldsymbol{\omega} / Q_{\text {effective }}=d \mathbf{r} d \boldsymbol{\omega} /\left(N_{\text {monomer }} V_{\text {eff }} \Omega_{\text {eff }}\right) .
$$
is

On the other hand, the probability of a random insertion

$$
d \mathbf{r} d \boldsymbol{\omega} / Q=d \mathbf{r} d \boldsymbol{\omega} / V_{\mathrm{box}} \Omega,
$$

where $\Omega=4 \pi$ for the model studied here.

Under these conditions, a conventional random position/ orientation would result in a dimer formation with probability

$$
Q_{\text {effective }} / Q=\left(N_{\text {monomer }} V_{\text {effective }} \Omega_{\text {effective }}\right) /\left(V_{\text {box }} \Omega\right) .
$$

For conventional simulations this ratio is very small and, therefore, the rate of creation or destruction of bonds very slow.

As shown schematically in Fig. 4, we can enhance this ratio by directing insertions to one of these effective positions/orientations (insertion with bond formation). More specifically, we select an insertion into one of these $Q_{\text {effective }}$ positions with probability $\xi$ and into one of the $Q-Q_{\text {effective }}$ positions with probability $1-\xi$. If we regard the effective positions/orientations as the $j_{a}$ states and the other positions as the $j_{b}$ states of our earlier analysis, then

$$
\begin{aligned}
& T_{i j_{a}}^{\text {insert }}=\frac{1}{N+1} \frac{\xi}{Q_{\text {effective }}} \\
& T_{i j_{b}}^{\text {insert }}=\frac{1}{N+1} \frac{1-\xi}{Q-Q_{\text {effective }}},
\end{aligned}
$$

where the $1 /(N+1)$ factor results from the indistinguishability of the inserted particle. ${ }^{13}$ The deletion of a particle takes place in the usual manner, i.e., by selecting any particle regardless of whether it is a monomer or a member of a dimer; we therefore have

$$
T_{i j_{a, b}}^{\text {delete }}=\frac{1}{N+1} \text {. }
$$

A simpler form of the proposed algorithm can be obtained by substituting Eqs. (39) and (40) into Eq. (32) and by considering the implementation of the above algorithm in an open ensemble such as the grand canonical. A microstate with $N$ particles located in the $d \mathbf{r}^{N} d \boldsymbol{\omega}^{N}$ differential hyper

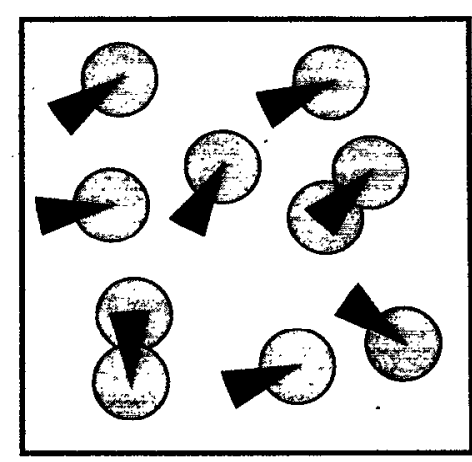

FIG. 4. Total exclusive volume and effective area for insertion with dimer formation. 
volume of a box having volume $V$, at temperature $T$ and with chemical potential $\mu$, occurs with probability

$$
p_{i}=\frac{1}{\Xi} \frac{z^{N}}{N !} \exp \left(-\beta U_{N}\right) d \mathbf{r}^{N} d \boldsymbol{\omega}^{N},
$$

where

$$
\Xi=\sum_{N} \frac{\lambda^{N}}{N ! \Lambda^{3 N}} \int_{Q} \exp (-\beta U) d \mathbf{r}^{N} d \omega^{N}
$$

is the partition function for the grand canonical ensemble ${ }^{14}$ and where $z=\exp (\mu k T) / \Lambda^{3}$ and $\lambda=\exp (\mu k T)$.

By means of an insertion, we propose a move from a state with $N$ particles into a state having $N+1$ particles and the same temperature, volume, and chemical potential. We then have

$$
\begin{aligned}
& T_{\text {bond }}^{\text {insertion }}=\frac{\xi}{N+1} \frac{d \mathbf{r} d \boldsymbol{\omega}}{Q_{\text {eff }}}=f_{\text {bond }}\left(\frac{1}{N+1} \frac{d \mathbf{r} d \boldsymbol{\omega}}{Q}\right), \\
& T_{\text {bond }}^{\text {deletion }}=\frac{1}{N+1}, \\
& T_{\text {free }}^{\text {inserion }}=\frac{1-\xi}{N+1} \frac{d \mathbf{r} d \boldsymbol{\omega}}{Q-Q_{\text {eff }}}=f_{\text {frec }}\left(\frac{1}{N+1} \frac{d \mathbf{r} d \boldsymbol{\omega}}{Q}\right), \\
& T_{\text {free }}^{\text {deletion }}=\frac{1}{N+1},
\end{aligned}
$$

where

$$
\begin{aligned}
& f_{\mathrm{bond}}^{\mathrm{insert}}=\xi \frac{Q}{Q_{\mathrm{eff}}}, \\
& f_{\mathrm{free}}^{\text {insert }}=(1-\xi) \frac{Q}{Q-Q_{\mathrm{eff}}},
\end{aligned}
$$

and where a parameter $f$ measures the bias introduced into the system. Subscript "free" denotes the parameters corresponding to an insertion without bond formation, and subscript "bond" denotes parameters corresponding to an insertion with bond formation.

From microscopic reversibility we have

$$
\begin{aligned}
& p_{N} T_{\text {bond }}^{\text {insert }} A_{\text {bond }}^{\text {insert }}=p_{N+1} T_{\text {bond }}^{\text {delete }} A_{\text {bond }}^{\text {delete }}, \\
& p_{N} T_{\text {free }}^{\text {insert }} A_{\text {free }}^{\text {insert }}=p_{N+1} T_{\text {free }}^{\text {delete }} A_{\text {free }}^{\text {delete }},
\end{aligned}
$$

which after using Eqs. (41) and (43) give

$$
\begin{aligned}
q_{\text {bond }}^{\text {insert }} & =\frac{A_{\text {bond }}^{\text {insert }}}{A_{\text {bond }}^{\text {delete }}}, \\
& =\frac{T_{\text {bond }}^{\text {delete }} p_{N+1}}{T_{\text {bond }}^{\text {insert }} p_{N}}, \\
& =f_{\text {bond }}^{\text {inscrt }} \frac{z V \Omega}{N+1} \exp (-\beta \Delta U), \\
q_{\text {free }}^{\text {insert }} & =f_{\text {free }}^{\text {insert }} \frac{z V \Omega}{N+1} \exp (-\beta \Delta U)
\end{aligned}
$$

which translates into accepting an attempt for insertion with or without dimer formation with probability $\min \left(1, q_{\text {bond }}^{\text {insert }}\right)$ or $\min \left(1, q_{\text {free }}^{\text {insert }}\right)$, respectively.

The governing equations for the deletion of a particle are very similar to those for insertion except that the process now starts with $N$ particles and goes into a state with $N-1$ particles. It can be shown that

$$
\begin{aligned}
& q_{\text {bond }}^{\text {delete }}=\frac{1}{f_{\text {bond }}^{\text {delete }}} \frac{N}{z V \Omega} \exp (-\beta \Delta U), \\
& q_{\text {free }}^{\text {delete }}=\frac{1}{f_{\text {free }}^{\text {delete }}} \frac{N}{z V \Omega} \exp (-\beta \Delta U),
\end{aligned}
$$

where

$$
\begin{aligned}
& f_{\text {bond }}^{\text {delete }}=\frac{1}{\xi} \frac{Q_{\text {eff }}}{Q}=\frac{1}{f_{\text {bond }}^{\text {insert }}}, \\
& f_{\text {free }}^{\text {delete }}=\frac{1}{1-\xi} \frac{Q-Q_{\text {eff }}}{Q}=\frac{1}{f_{\text {free }}^{\text {insert }}} .
\end{aligned}
$$

In that case an attempt to delete a particle will be accepted with probability $\min \left(1, q_{\text {free }}^{\text {delete }}\right)$, or $\min \left(1, q_{\text {bond }}^{\text {delete }}\right)$, depending on whether the randomly particle chosen for deletion is a monomer or a member of a dimer. As the above equations suggest, no major modifications to the originalGibbs ensemble algorithm are required and the implementation of the biased method is remarkably simple. Note that a value of parameter $\xi$ close to zero reduces the creation of dimers during insertion; a value close to 1 leads to insertions that result almost exclusively in the creation of new dimers. A value of $\xi=Q / Q_{\text {eff }}$ removes the bias and leads to conventional grand canonical ensemble sampling.

The extension to the Gibbs ensemble is done within the same framework and differs from from the conventional Gibbs-ensemble algorithm in the insertion/deletion step. More specifically, that step is replaced by the following elementary moves:

(1) With equal probability, choose the phase that receives a particle (e.g., phase 2) and the phase that donates the particle (e.g., phase 1).

(2a) With probability $\xi$, insert a monomer in any of the possible positions/orientations of phase 2 that result in the formation of a dimer. Then compute $f^{(2)}$ as $f_{\text {bond }}^{(2)}$ given below. Or

(2b) with probability $1-\xi$ insert in any position/orientation that does not create a new dimer. Evaluate $f^{(2)}$ as $f_{\text {free }}^{(2)}$.

(3) Pick at random a particle from phase 1 for deletion and determine whether the particle is part of a dimer or whether it is a monomer. Then evaluate $f^{(1)}$ as either $f_{\text {free }}^{(1)}$ (if particle was a monomer) or $f_{\text {bond }}^{(1)}$ (particle was part of a dimer).

(4) Accept the proposed particle transfer with probability

$$
\begin{aligned}
& A=\min (1, q), \\
& q=\exp (-\beta \Delta \Gamma),
\end{aligned}
$$




$$
\begin{aligned}
\Delta \Gamma= & {\left[\Delta U_{(1)}+\Delta U_{(2)}-\frac{1}{\beta} \ln \frac{V_{(2)}}{V_{(1)}}-\frac{1}{\beta} \ln \frac{N_{(1)}}{N_{(2)}+1}\right] } \\
& -\frac{1}{\beta} \ln \frac{f_{(2)}}{f_{(1)}}
\end{aligned}
$$

where

$$
\begin{aligned}
& f_{\text {bond }}^{(1)}=\xi \frac{Q^{(1)}}{Q_{\text {eff }}^{(1)}}, \\
& f_{\text {free }}^{(1)}=(1-\xi) \frac{Q^{(1)}}{Q^{(1)}-Q_{\text {eff }}^{(1)}}, \\
& f_{\text {bond }}^{(2)}=\frac{1}{\xi} \frac{Q_{\text {eff }}^{(2)}}{Q^{(2)}}, \\
& f_{\text {free }}^{(2)}=\frac{1}{(1-\xi)} \frac{Q^{(2)}-Q_{\text {eff }}^{(2)}}{Q^{(2)}} .
\end{aligned}
$$

\section{RESULTS AND DISCUSSION}

\section{A. Simulation details}

We have used our newly proposed BBMC method to simulate vapor-liquid equilibria for associating fluids modeled as Lennard-Jones spheres with an off-center square well attraction site. Following Chapman's initial work, we have used a value of $27^{\circ}$ for the angle $\theta_{c}$, and a value of $1 \sigma$ for the $r_{c}$ distance (see Fig. 1). We should emphasize that for this specific model, the value of $l$, which corresponds to the distance from the center of the sphere to the association site, does not intervene explicitly in any of the calculations. The BBMC method has been used to obtain the coexistence curve for various association strengths.

The simulations were performed on a system of 512 particles. In our computer simulations, we used a "bonding" array to test "steric incompatibilities;" in this array, every molecule has an integer with the value of -1 if the molecule is free (monomer) or with the number of its "associate" if it is part of a dimer. During all of our simulations, no attraction site was found to be bonded to more than one other site. The maximum displacement, the maximum angle rotation, and the maximum volume changes where adjusted to yield an acceptance ratio of about 50\%. As explained in the Appendix, we used Jansoone's method to rotate the particle and to make the special insertion necessary for the biased transfer step. All the simulations were started from an FCC structure and were equilibrated for 5000 Monte Carlo cycles without any attempt for particle transfers. Every MC cycle consists of moving all the particles, changing the volume of the boxes, and transferring particles. An additional $15 \mathrm{~K} \mathrm{MC}$ cycles were used for further equilibration with particle transfers followed by $50-100 \mathrm{~K}$ MC steps for statistical averaging. Blocks of $1 \mathrm{~K} \mathrm{MC}$ steps were used and the standard deviation of their mean was used as an estimate for the uncertainty of the average value. ${ }^{15}$ Finally, the number of transfer attempts was varied from 10 to 50 as the system was cooled to lower temperatures.

We have investigated different values of parameter $\xi$, which controls the degree of biasing introduced into the sys-

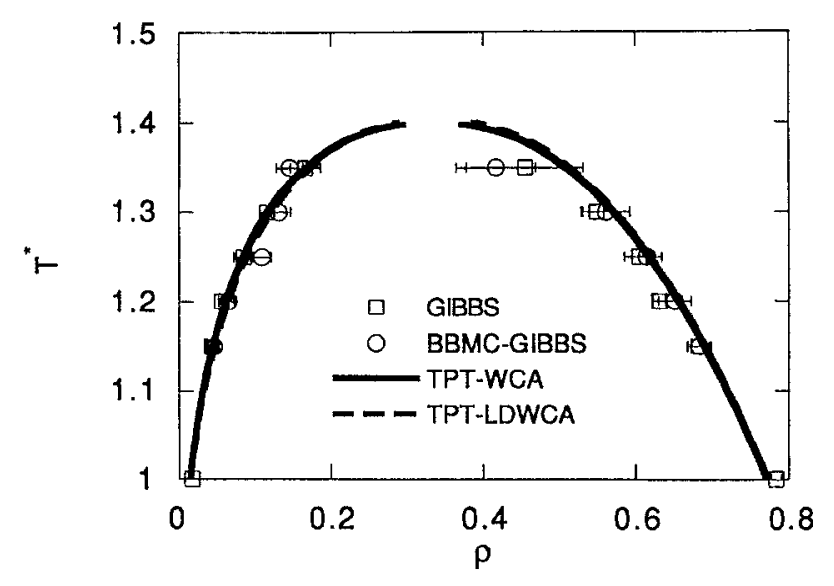

FIG. 5. Temperature vs density coexistence curve for LJ associating fluids with $\epsilon_{\mathrm{SW}}=8 \epsilon_{\mathrm{LJ}}$.

tem. Table I gives simulation results for values ranging between $10 \%$ and $90 \%$. Even though the final results are independent of $\xi$, values close to $0 \%$ or close to $100 \%$ are not recommended because they lead to inefficient sampling of dimer formation or monomer equilibration, respectively. For simulations of fluids with $\epsilon_{\mathrm{SW}}>10 \epsilon_{\mathrm{L}}$, only values between $30 \%-70 \%$ were used. The value of the effective volume/ orientation required for Eqs. (50)-(53) was calculated exactly by carrying out the necessary integrations (analytically) as explained in the Appendix $b$.

\section{B. Simulation of saturation properties}

Figure 5 shows the saturation density for a fluid with $\epsilon_{\mathrm{SW}}=8 \epsilon_{\mathrm{LJ}}$ calculated using both the conventional Gibbs ensemble method and the newly proposed BBMC method. For comparison, we also report the values of the saturation properties as predicted from the thermodynamic perturbation theory presented earlier using both the WCA approximation over the full density range (denoted TPT-WCA) and the semiempirical formula proposed by Johnson and Gubbins ${ }^{7}$

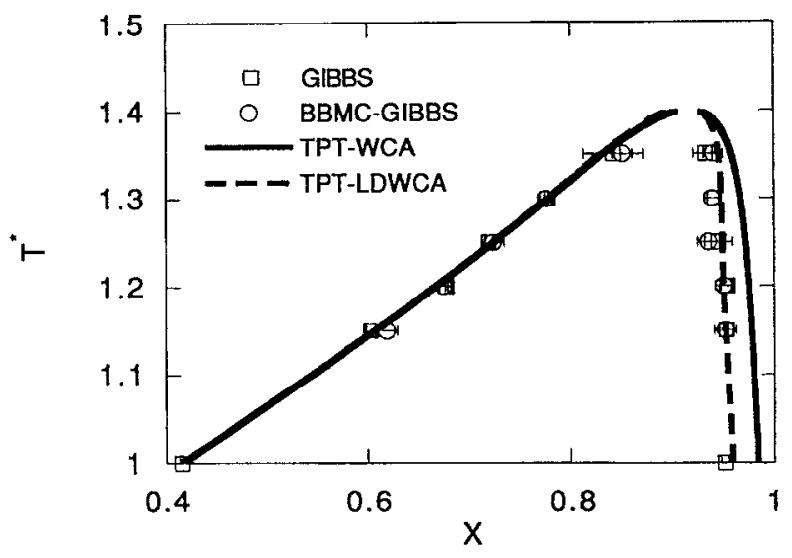

FIG. 6. Temperature vs monomer-fraction diagram for saturated $\mathrm{LJ}$ associating fluids with $\epsilon_{\mathrm{SW}}=8 \epsilon_{\mathrm{LJ}}$. 
TABLE I. Comparison between theory (TPT), Conventional Gibbs results (Gibbs) and bond-bias MC in the Gibbs ensemble (BBMC-Gibbs) results for $\epsilon_{\mathrm{SW}}=8 \epsilon_{\mathrm{LJ}}$

\begin{tabular}{|c|c|c|c|c|c|c|c|c|}
\hline Method & $T^{*}$ & $\xi(\%)$ & $\rho_{v}^{*}$ & $\rho_{l}^{*}$ & $U_{v}^{*}$ & $U_{l}^{*}$ & $x_{v}^{*}$ & $x_{i}^{*}$ \\
\hline TPT-WCA & 1.00 & & 0.015 & 0.772 & -0.18 & -7.30 & 0.985 & 0.416 \\
\hline TPT-LDWCA & & & 0.015 & 0.771 & -0.28 & -7.29 & 0.959 & 0.416 \\
\hline Gibbs & 1.00 & & 0.017 & $0.783(7)$ & $-0.33(2)$ & $-7.34(7)$ & 0.952 & $0.416(2)$ \\
\hline TPT-WCA & 1.15 & & 0.045 & 0.689 & -0.45 & -5.99 & 0.979 & 0.607 \\
\hline TPT-LDWCA & & & 0.046 & 0.689 & -0.56 & -5.99 & 0.953 & 0.607 \\
\hline Gibbs & 1.15 & & $0.043(5)$ & $0.681(14)$ & $-0.53(6)$ & $-5.95(12)$ & $0.953(6)$ & $0.603(4)$ \\
\hline \multirow[t]{5}{*}{ BBMC-Gibbs } & 1.15 & 10 & $0.046(3)$ & $0.679(14)$ & $-0.55(4)$ & $-5.86(15)$ & $0.954(6)$ & $0.626(17)$ \\
\hline & & 30 & $0.048(5)$ & $0.686(11)$ & $-0.57(6)$ & $-5.95(9)$ & $0.952(7)$ & $0.613(11)$ \\
\hline & & 50 & $0.046(5)$ & $0.683(15)$ & $-0.56(7)$ & $-5.91(13)$ & $0.952(10)$ & $0.619(10)$ \\
\hline & & 70 & 0.051 (5) & $0.692(13)$ & $-0.62(7)$ & $-5.99(13)$ & $0.948(6)$ & $0.614(14)$ \\
\hline & & 90 & $0.046(6)$ & $0.682(16)$ & $-0.55(8)$ & $-5.94(13)$ & $0.953(7)$ & $0.609(3)$ \\
\hline TPT-WCA & 1.20 & & 0.062 & 0.656 & -0.59 & -5.55 & 0.976 & 0.667 \\
\hline TPT-LDWCA & & & 0.064 & 0.656 & -0.70 & -5.54 & 0.951 & 0.667 \\
\hline Gibbs & 1.20 & & $0.056(4)$ & 0.631 & $-0.61(5)$ & $-5.35(6)$ & $0.954(5)$ & 0.678 (1) \\
\hline \multirow[t]{5}{*}{ BBMC-Gibbs } & 1.20 & 10 & $0.063(9)$ & $0.644(19)$ & $-0.68(11)$ & $-5.43(18)$ & $0.952(8)$ & $0.678(13)$ \\
\hline & & 30 & $0.066(7)$ & $0.646(13)$ & $-0.72(8)$ & $-5.45(11)$ & $0.950(8)$ & $0.677(7)$ \\
\hline & & 50 & $0.066(5)$ & $0.649(17)$ & $-0.72(6)$ & $-5.46(13)$ & $0.950(6)$ & $0.676(9)$ \\
\hline & & 70 & $0.065(8)$ & $0.651(21)$ & $-0.70(9)$ & $-5.49(17)$ & $0.951(8)$ & $0.675(3)$ \\
\hline & & 90 & $0.074(9)$ & $0.654(23)$ & $-0.81(11)$ & $-5.51(17)$ & $0.943(11)$ & 0.671 (9) \\
\hline TPT-WCA & 1.25 & & 0.085 & 0.618 & -0.77 & -5.08 & 0.973 & 0.724 \\
\hline TPT-LDWCA & & & 0.088 & 0.618 & -0.88 & -5.08 & 0.949 & 0.723 \\
\hline Gibbs & 1.25 & & $0.085(13)$ & $0.605(19)$ & $-0.86(15)$ & $-5.01(17)$ & $0.946(13)$ & 0.719 (3) \\
\hline \multirow[t]{5}{*}{ BBMC-Gibbs } & 1.25 & 10 & $0.077(13)$ & $0.598(23)$ & $-0.75(13)$ & $-4.94(21)$ & $0.957(13)$ & $0.730(19)$ \\
\hline & & 30 & $0.109(12)$ & $0.615(19)$ & $-1.08(13)$ & $-5.06(14)$ & $0.936(10)$ & $0.724(7)$ \\
\hline & & 50 & $0.102(20)$ & $0.609(21)$ & $-1.02(22)$ & $-4.98(19)$ & $0.935(17)$ & $0.739(12)$ \\
\hline & & 70 & $0.096(20)$ & $0.605(28)$ & $-0.96(20)$ & $-4.98(22)$ & $0.938(8)$ & 0.730 \\
\hline & & 90 & $0.149(14)$ & $0.625(22)$ & $-1.43(14)$ & $-5.12(19)$ & $0.926(13)$ & $0.722(14)$ \\
\hline TPT-WCA & 1.30 & & 0.117 & 0.572 & -1.02 & -4.57 & 0.968 & 0.779 \\
\hline TPT-LDWCA & & & 0.120 & 0.572 & -1.12 & -4.58 & 0.949 & 0.778 \\
\hline Gibbs & 1.30 & & $0.116(10)$ & $0.550(21)$ & $-1.10(10)$ & $-4.44(16)$ & $0.942(7)$ & 0.778 \\
\hline \multirow[t]{4}{*}{ BBMC-Gibbs } & 1.30 & 10 & $0.131(16)$ & $0.562(31)$ & $-1.22(14)$ & $-4.53(26)$ & $0.939(7)$ & $0.776(13)$ \\
\hline & & 30 & $0.113(15)$ & $0.520(31)$ & $-1.06(13)$ & $-4.18(23)$ & $0.944(10)$ & $0.797(11)$ \\
\hline & & 50 & $0.128(18)$ & $0.535(42)$ & $-1.20(17)$ & $-4.30(33)$ & $0.938(10)$ & $0.789(7)$ \\
\hline & & 90 & $0.161(14)$ & $0.577(32)$ & $-1.52(13)$ & $-4.63(25)$ & $0.921(9)$ & 0.770 \\
\hline TPT-WCA & 1.35 & & 0.167 & 0.509 & -1.40 & -3.97 & 0.959 & 0.833 \\
\hline TPT-LDWCA & & & 0.170 & 0.512 & -1.47 & -3.99 & 0.946 & 0.831 \\
\hline Gibbs & 1.35 & & $0.145(15)$ & $0.482(44)$ & $-1.30(13)$ & $-3.81(32)$ & $0.942(11)$ & $0.831(18)$ \\
\hline BBMC-Gibbs & 1.35 & 10 & $0.145(17)$ & $0.417(52)$ & $-1.30(15)$ & $-3.35(38)$ & $0.941(9)$ & $0.851(13)$ \\
\hline
\end{tabular}

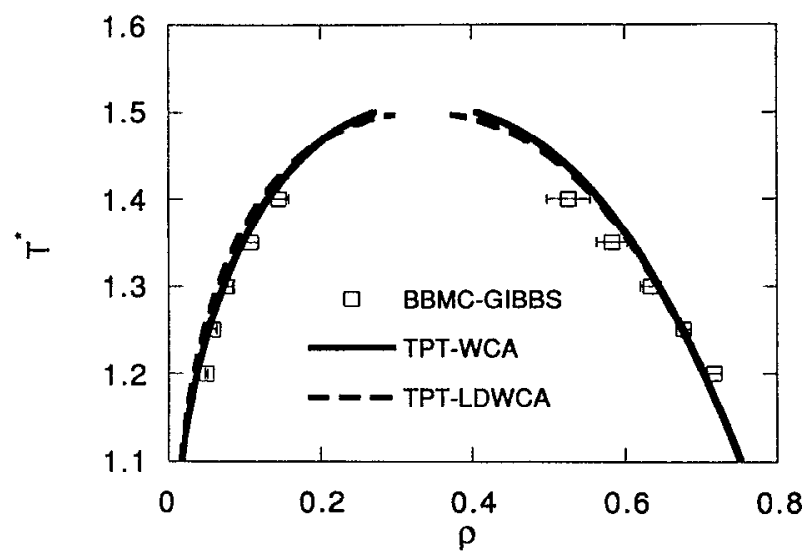

FIG. 7. Temperature vs density coexistence curve for $\mathrm{LJ}$ associating fluids with $\epsilon_{\mathrm{SW}}=10 \epsilon_{\mathrm{L}}$.

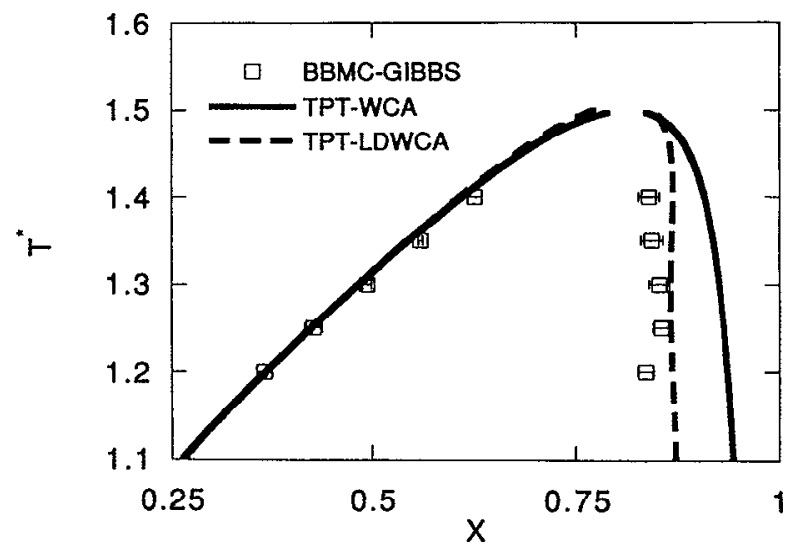

FIG. 8. Temperature vs monomer-fraction diagram for saturated LJ associating fluids with $\epsilon_{\mathrm{SW}}=10 \epsilon_{\mathrm{L}}$. 
TABLE II. Comparison between theory (TPT) and bond-bias MC in the Gibbs ensemble (BBMC-Gibbs) results for $\epsilon_{\mathrm{SW}}=10 \epsilon_{\mathrm{LJ}}$.

\begin{tabular}{|c|c|c|c|c|c|c|c|c|}
\hline Method & $T^{*}$ & $\rho_{v}^{*}$ & $\rho_{l}^{*}$ & $U_{v}^{*}$ & $U_{l}^{*}$ & $x_{v}^{*}$ & $x_{i}^{*}$ & $P^{*}$ \\
\hline TPT-WCA & 1.20 & 0.034 & 0.704 & -0.54 & -7.49 & 0.938 & 0.367 & 0.035 \\
\hline TPT-LDWCA & & 0.037 & 0.704 & -0.91 & $-7,49$ & 0.869 & 0.366 & 0.036 \\
\hline BBMC-Gibbs & & $0.050(2)$ & $0.718(10)$ & $-1.18(7)$ & $-7.55(13)$ & $0.837(10)$ & $0.365(10)$ & \\
\hline TPT-WCA & 1.25 & 0.048 & 0.676 & -0.66 & -7.03 & 0.933 & 0.424 & 0.048 \\
\hline TPT-LDWCA & & 0.053 & 0.676 & -1.02 & -7.03 & 0.867 & 0.423 & 0.050 \\
\hline BBMC-Gibbs & & $0.059(4)$ & $0.678(10)$ & $-1.14(8)$ & $-7.01(11)$ & $0.856(10)$ & $0.426(11)$ & \\
\hline TPT-WCA & 1.30 & 0.066 & 0.645 & -0.82 & -6.52 & 0.927 & 0.483 & 0.065 \\
\hline TPT-LDWCA & & 0.072 & 0.645 & -1.17 & -6.52 & 0.867 & 0.482 & 0.068 \\
\hline BBMC-Gibbs & & $0.077(7)$ & $0.634(14)$ & $-1.28(12)$ & $-6.41(14)$ & $0.853(12)$ & $0.493(5)$ & \\
\hline TPT-WCA & 1.35 & 0.090 & 0.608 & -1.02 & -5.98 & 0.920 & 0.545 & 0.085 \\
\hline TPT-LDWCA & & 0.097 & 0.609 & -1.34 & -5.99 & 0.868 & 0.544 & 0.089 \\
\hline BBMC-Gibbs & & $0.108(11)$ & $0.583(20)$ & $-1.55(15)$ & $-5.78(16)$ & $0.844(13)$ & $0.560(2)$ & \\
\hline TPT-WCA & 1.40 & 0.123 & 0.563 & -1.30 & -5.38 & 0.909 & 0.611 & 0.110 \\
\hline TPT-LDWCA & & 0.130 & 0.566 & -1.56 & -5.41 & 0.870 & 0.609 & 0.115 \\
\hline BBMC-Gibbs & & $0.145(13)$ & $0.526(28)$ & $-1.81(15)$ & $-5.11(22)$ & $0.840(13)$ & $0.627(7)$ & \\
\hline
\end{tabular}

(denoted TPT-LDWCA). Both simulation methods give results that are within the error estimates for the entire range of saturation densities; Fig. 6 shows the monomer fraction for a fluid with $\epsilon_{\mathrm{Sw}}=8 \epsilon_{\mathrm{LJ}}$; again, there is excellent agreement between the conventional Gibbs ensemble simulation results and those of our newly proposed method (BBMC-Gibbs). Given that BBMC biases the formation/destruction of bonds, the fact that both methods yield identical monomer fractions in both phases provides some reassurance about the correctness of the new approach. Figures 5 and 6 also show the results of the TPT-WCA and TPT-LDWCA calculations. Agreement between theory and simulation is satisfactory. Table I gives exact simulation values (along with their error estimates) and TPT predictions.

In Table II we report results of simulations and the TPT calculations for a fluid interacting with a slightly stronger association potential. A value of $\epsilon_{S W}=10 \epsilon_{\mathrm{LJ}}$ was used for these simulations. Our simulation results appear to be in good agreement with those reported by Johnson and Gubbins. $^{7}$ Figures 7 and 8 show simulated and theoretical saturation densities and the fraction of monomers in the co-

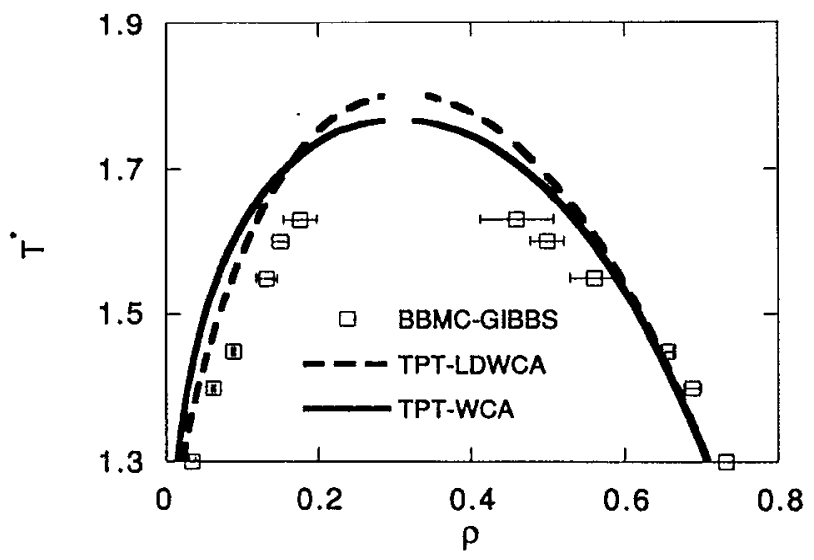

FIG. 9. Temperature vs density coexistence curve for $\mathrm{LJ}$ associating fluids with $\epsilon_{\text {Sw }}=15 \epsilon_{\mathrm{J}}$. existing phases, respectively. The fact that the theory overestimates the critical point is not surprising and is inherent to any mean field treatment. For temperatures below $T^{*}=1.45$, however, we obtain good agreement between simulated and theoretical orthobaric densities. Unfortunately, this good agreement is somewhat deceiving in that it disguises the fact that association is not predicted accurately; as Fig. 8 illustrates, the simulated fraction of monomers in the vapor phase is well below that predicted by the WCA version of the theory. Note, however, that the TPT-LDWCA version leads to better agreement with simulation. These findings are consistent with those of Johnson and Gubbins. ${ }^{7}$

As shown in Fig. 9 the tendency of the theory to underestimate critical properties becomes more pronounced for higher association strengths. For associating fluids interacting with a square well potential equal to $15 \epsilon_{\mathrm{LJ}}$, the theory largely over predicts the critical temperature. At lower temperatures, however, it predicts saturated liquid densities in fair agreement with the results of our simulations. The underestimation of the vapor phase association by both versions of the theory is much more significant for these fluids;

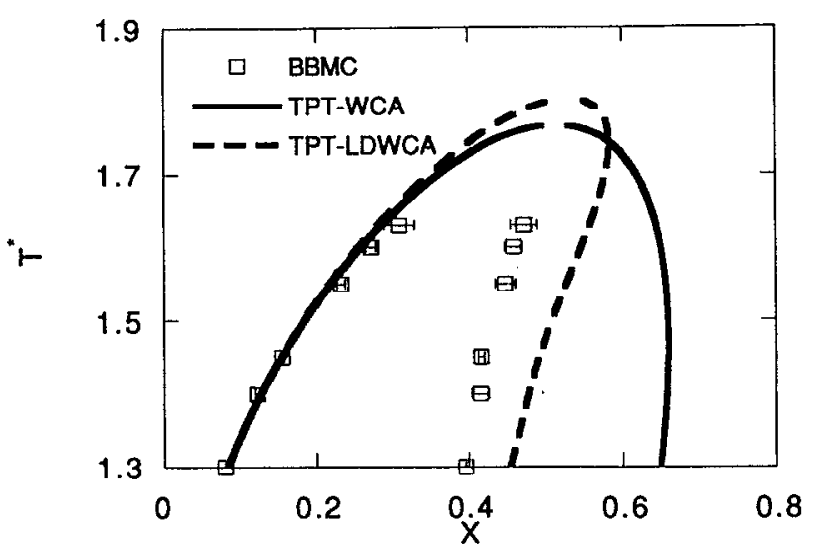

FIG. 10. Temperature vs monomer-fraction diagram for saturated $\mathrm{LJ}$ associating fluids with $\epsilon_{\mathrm{SW}}=15 \epsilon_{\mathrm{LJ}}$. 
TABLE III. Comparison between theory (TPT) and bond-bias MC in the Gibbs ensemble (BBMC-Gibbs) results for $\epsilon_{\mathrm{SW}}=15 \epsilon_{\mathrm{LJ}}$.

\begin{tabular}{|c|c|c|c|c|c|c|c|c|}
\hline Method & $T^{*}$ & $\rho_{v}^{*}$ & $\rho_{l}^{*}$ & $U_{v}^{*}$ & $U_{l}^{*}$ & $x_{v}^{*}$ & $x_{l}^{*}$ & $p^{*}$ \\
\hline TPT-LDWCA & & 0.022 & 0.708 & -4.08 & -10.91 & 0.455 & 0.084 & 0.019 \\
\hline TPT-WCA & 1.40 & 0.028 & 0.667 & -2.46 & -10.28 & 0.658 & 0.128 & 0.030 \\
\hline TPT-LDWCA & & 0.041 & 0.668 & -4.05 & -10.29 & 0.478 & 0.127 & 0.037 \\
\hline BBMC-Gibbs & & $0.062(2)$ & $0.688(11)$ & $-4.62(16)$ & $-10.45(12)$ & $0.416(11)$ & 0.123 & \\
\hline BBMC-Gibbs & & $0.088(2)$ & $0.656(7)$ & $-4.79(6)$ & $-9.97(6)$ & $0.417(4)$ & $0.155(2)$ & \\
\hline TPT-WCA & 1.55 & 0.067 & 0.590 & -2.73 & -9.07 & 0.657 & 0.219 & 0.069 \\
\hline TPT-LDWCA & & 0.086 & 0.595 & -3.92 & -9.11 & 0.528 & 0.217 & 0.083 \\
\hline BBMC-Gibbs & & $0.132(14)$ & $0.561(31)$ & $-4.83(20)$ & $-8.81(23)$ & $0.449(13)$ & $0.232(5)$ & \\
\hline TPT-WCA & 1.60 & 0.088 & 0.557 & -2.91 & -8.57 & 0.650 & 0.259 & 0.089 \\
\hline TPT-LDWCA & & 0.106 & 0.565 & -3.89 & -8.64 & 0.547 & 0.255 & 0.104 \\
\hline BBMC-Gibbs & & $0.150(11)$ & $0.500(22)$ & $-4.87(21)$ & $-8.16(17)$ & $0.459(11)$ & $0.272(6)$ & \\
\hline
\end{tabular}

even the TPT-LDWCA version of the theory leads only to semiquantitative agreement with simulation results. Figure 10 shows that, in addition to providing low estimates to the monomer fraction, the theory predicts vapor orthobaric densities that are lower than those obtained from simulations. Table III gives the exact simulation results and the predictions of the theory.

A comparison of Figs. 8 and 10 shows that, as the association strength increases, the degree of association in both phases also increases, thereby shifting the monomer fraction saturation curve to the left. We must emphasize that any attempt to simulate these fluids using the conventional Gibbs ensemble method would fail. In contrast, the BBMC method is successful in converging to equilibrium within a few thousand MC cycles. As shown in Figs. 9 and 10, these fluids exhibit significant association; therefore our choice of $\xi=50 \%$ does not contradict the fact that our main focus should be that of sampling dimer creation/destruction.

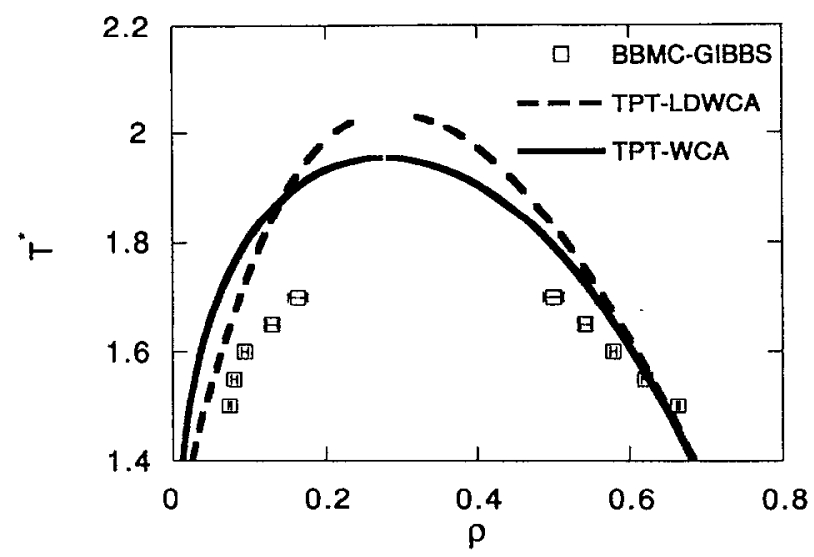

FIG. 11. Temperature vs density coexistence curve for $\mathrm{LJ}$ associating fluids with $\epsilon_{\mathrm{SW}}=20 \epsilon_{\mathrm{LJ}}$.
The BBMC method was also used for the study of vapor-liquid equilibria of very strongly associating fluids. Strong associations were modeled by using a value of $20 \epsilon_{\mathrm{LJ}}$ for the square-well interaction. As shown in Figs. 11 and 12, the TPT-WCA version of the theory is able to predict orthobaric densities only semiquantitatively and only for temperatures well below the critical point (see Table IV also). The TPT-LDWCA version gives slightly better estimates for the monomer fraction of the vapor phase but it leads to an association curve whose shape (a "banana" shape) is inconsistent with that observed in our simulations. As expected, the simulated degree of association in the vapor phase is very high; the theory, however, fails to predict this vapor-phase dimerization accurately. This is an important shortcoming, particularly when we consider the fact that real associating fluids such as carboxylic acids are known to dimerize significantly in the vapor phase and that the vapor-

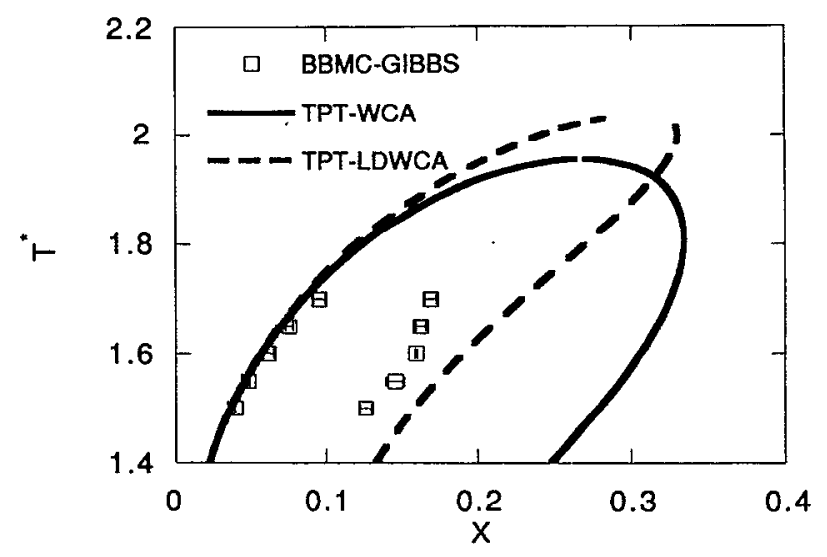

FIG. 12. Temperature vs monomer-fraction diagram for saturated $\mathrm{LJ}$ associating fluids with $\epsilon_{\mathrm{SW}}=20 \epsilon_{\mathrm{LJ}}$. 
TABLE IV. Comparison between theory (TPT) and bond-bias MC in the Gibbs ensemble (BBMC-Gibbs) results for $\epsilon_{\mathrm{SW}}=20 \epsilon_{\mathrm{LJ}}$.

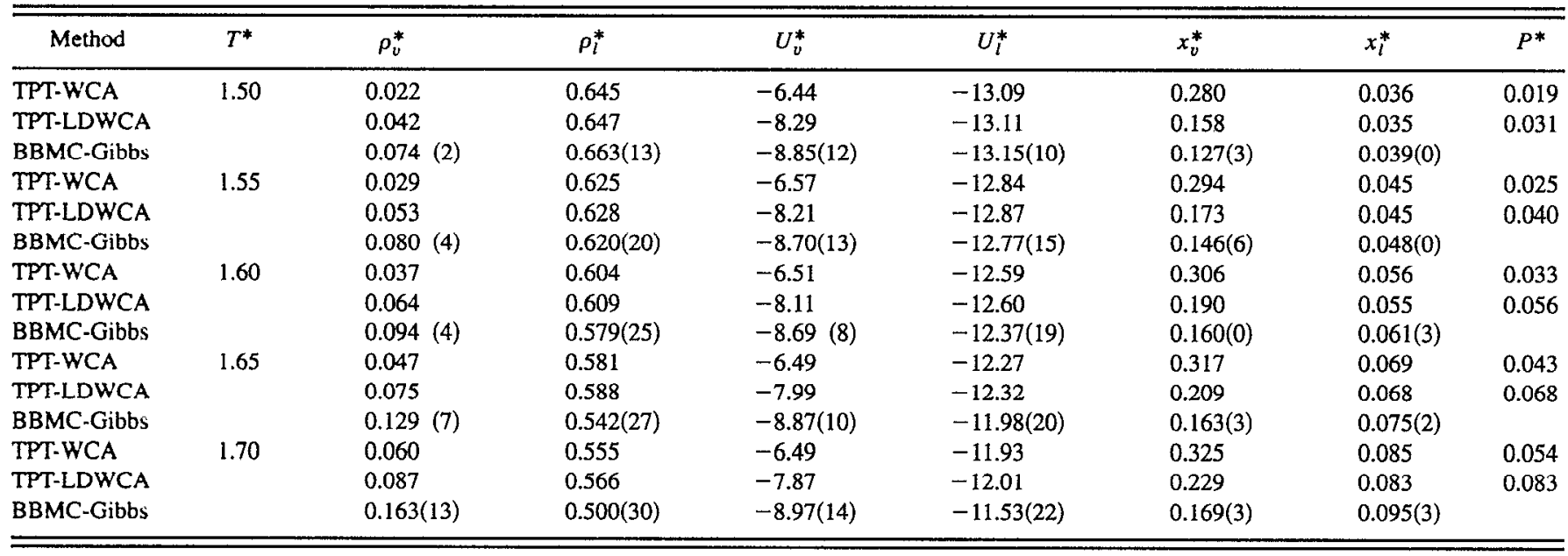

phase dimerization is largely responsible for the strong nonidealities of such gases.

To illustrate the values of the association strength required to model real fluids, we have also attempted to describe the thermodynamic properties of acetic acid using the theory discussed in this work. While acetic acid forms two hydrogen bonds when it forms a dimer (see Fig. 13), for simplicity we assume that a single attraction site provides a reasonable description of the fluid. Table $\mathrm{V}$ reports the parameters obtained after fitting experimental saturation densities to theoretical predictions using the TPT-WCA version. The fitting was done for various association strengths; the fourth column in this table shows the ratio of the mean square error with respect to the error obtained at $\epsilon_{\mathrm{SW}}=20 \epsilon_{\mathrm{LJ}}\left[\chi^{2} / \chi_{20}^{2}\right]$. The experimental values for acetic acid were obtained from Ref. 16. Figure 14 shows that the theory describes the experimental coexistence curve with good accuracy, particularly in regions remote from the critical point. For estimation of these parameters only saturation data between $150-300^{\circ} \mathrm{C}$ were used (we do not expect this model to be quantitative in the critical region).

Figure 15 shows the theoretical and experimental temperature dependence of the saturation pressure. For high densities, the theory overestimates the experimental pressure probably because a spherical model does not have the more appropriate "dumbbell" shape of acetic acid.

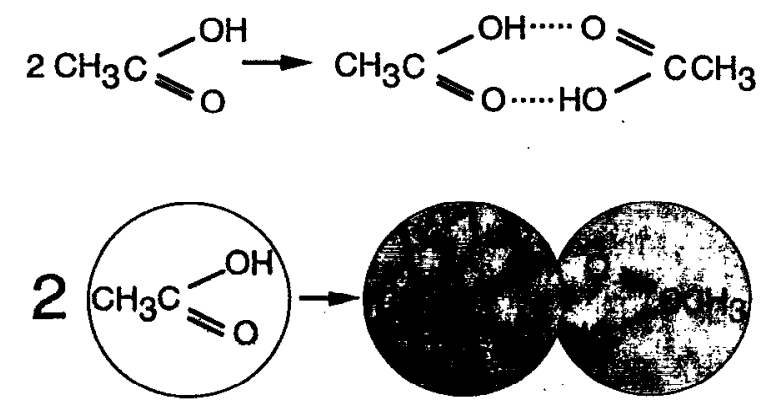

FIG. 13. Dimerization of acetic acid.
Figure 16 shows that there is good agreement between theory and experiment for the latent heat of vaporization over the region of interest, indicating that an attractive energy of $20 \epsilon_{\mathrm{LJ}}$ describes reasonably well the energetics of acetic acid. As mentioned earlier for the saturation pressure, better agreement with experiment could be achieved by using more detailed molecular models for acetic acid (e.g., a dumbbell with two attraction sites); such studies, however, are beyond the scope of this work. In this paper we only wish to point out that high associating strengths (e.g., $20 \epsilon_{\mathrm{IJ}}$ ) are not of a mere academic interest but are actually necessary to describe quantitatively the thermodynamic properties of common associating fluids such as carboxylic acids.

The results shown in Figs. 11 and 12 for a strength of $20 \epsilon_{\mathrm{LJ}}$ clearly illustrate that a first order perturbation theory is unable to provide a satisfactory description of phase equilibria for strongly associating fluids. That earlier studies did not investigate the accuracy of TPT for high association strengths is probably due to the fact that appropriate molecular simulation techniques were not available. To a large extent, the limitations of the theory have only become apparent in light of our newly acquired ability to simulate such fluids with the BBMC method.

At high association strengths it is reasonable to expect a first order perturbation theory to be inadequate. Deviations from the reference system (a $\mathrm{LJ}$ fluid, in this case) become increasingly important as the association strength increases; eventually, first order terms are not sufficient and it is nec-

TABLE V. Model parameters for acetic acid.

\begin{tabular}{lccc}
\hline$\epsilon_{\mathrm{SW}}$ & $\begin{array}{c}\epsilon_{\mathrm{J}} \\
(\mathrm{K})\end{array}$ & $\begin{array}{c}\sigma_{\mathrm{LJ}} \\
(\AA)\end{array}$ & $\begin{array}{c}\chi^{2} \\
\chi^{2} / \chi_{20}^{2}\end{array}$ \\
\hline 10 & 408.638 & 4.444 & 4.945 \\
15 & 348.378 & 4.362 & 2.253 \\
16 & 340.108 & 4.343 & 1.846 \\
17 & 333.028 & 4.326 & 1.523 \\
18 & 326.985 & 4.311 & 1.277 \\
19 & 321.972 & 4.298 & 1.104 \\
20 & 317.709 & 4.286 & 1.000 \\
\hline
\end{tabular}




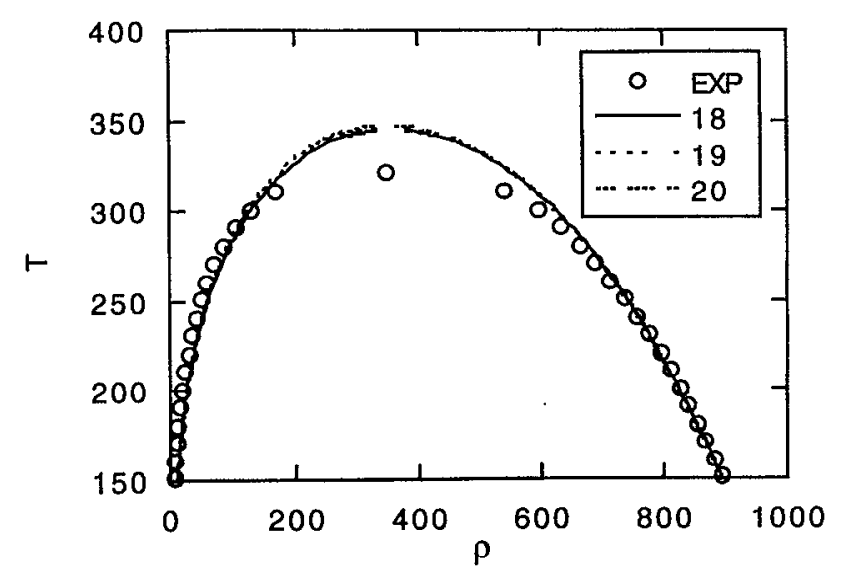

FIG. 14. Temperature vs density coexistence curve for acetic acid. The open symbols show experimental data. The lines show results of the theory for several association strengths. $\left(T\left[{ }^{\circ} \mathrm{C}\right]\right.$ and $\left.\rho\left[\mathrm{Kg} / \mathrm{m}^{3} \times 10^{3}\right]\right)$.

essary to go to second order perturbation terms in the expansion of the Helmholtz free energy. These second order terms require the use of three-body correlation functions. To reduce these terms to a tractable form, several approximations must be made; we expect our BBMC method to provide a valuable tool for testing such approximations and for the development of more accurate theories for strongly associating fluids.

\section{CONCLUSIONS}

We have presented a new Monte Carlo method which permits a preferential sampling of subregions of configuration space. The BBMC method, which requires minor changes to conventional MC algorithms, can be used in conjunction with any technique used to simulate open systems. As shown in this work, it provides an efficient tool for simulating the thermodynamic properties, including phase equilibria, of fluids that interact with rapidly varying potentials.

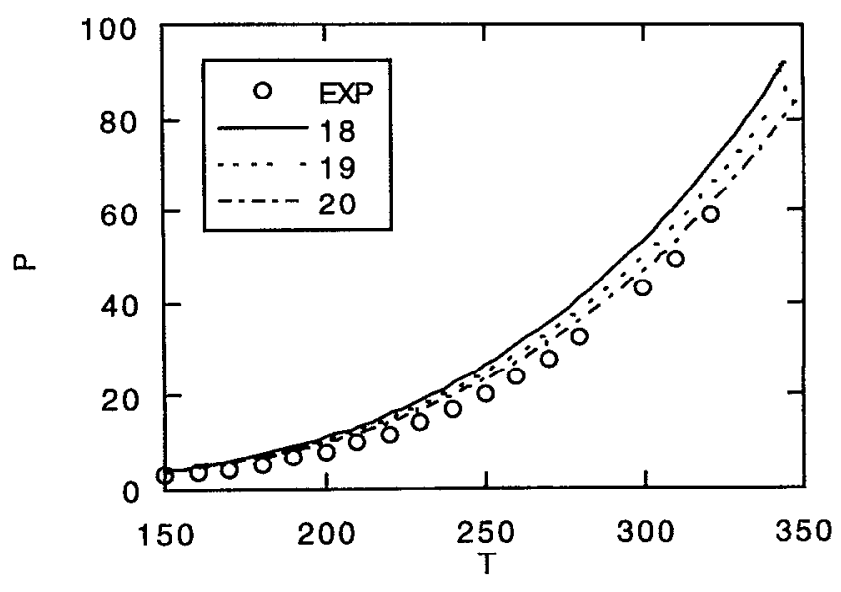

FIG. 15. Saturation pressure diagram for acetic acid. Theory and experimental results. ( $P[$ bar $]$ and $T\left[{ }^{\circ} \mathrm{C}\right]$ ).

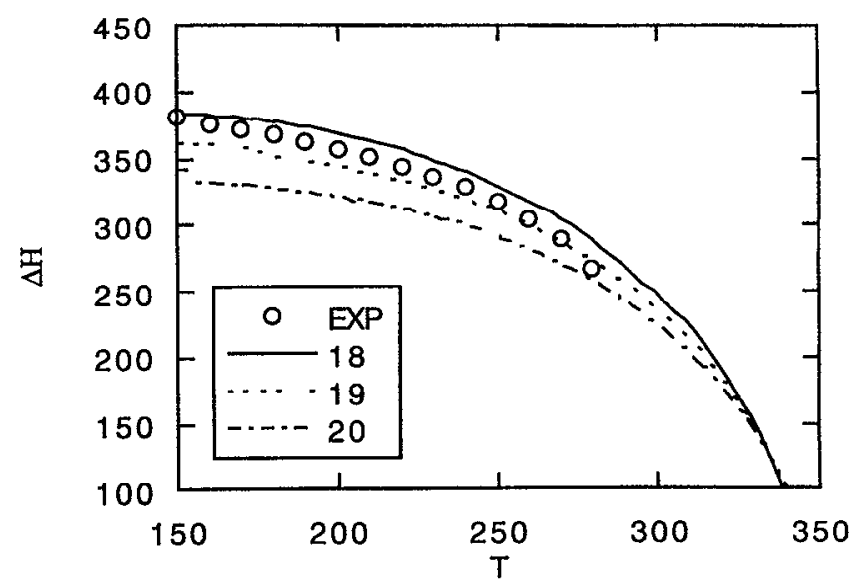

FIG. 16. Latent heat of vaporization diagram for acetic acid. Theory and experimental results. $\left(\Delta H[\mathrm{Kg} / \mathrm{KJ}]\right.$ and $\left.T\left[{ }^{\circ} \mathrm{C}\right]\right)$.

Here we have applied the BBMC method to simulate vapor-liquid equilibria for pure Lennard-Jones associating fluids interacting through square-well potentials of varying strengths. The exact results of our simulations indicate that the simple first order thermodynamic perturbation theory gives accurate predictions only for low to moderate association strengths. For higher strengths, the theory underpredicts the extent of association in the vapor phase. This deficiency is severe for association strengths similar to those required to model real associating fluids (e.g., acetic acid). Our simulations show that the structure of strongly associating fluids is significantly different from that of the LJ reference fluid, thereby leading to deviations in the free energy that cannot be accounted for by first order perturbation terms only. Clearly, higher order terms in the Helmholtz free energy expansion are necessary for more accurate results.

Aside from the shortcomings discussed above, however, the theory provides a solid foundation for building and testing more accurate molecular models of real associating fluids. We have shown here that a simple fit of the vapor-liquid coexistence curve of acetic acid is able to capture both the phase equilibria and the energetics of associating fluids. Better agreement between theory and experiment can be expected from a formalism that relies on a molecular model that mimics more closely the real molecular structure of carboxylic acids and that incorporates second order thermody-

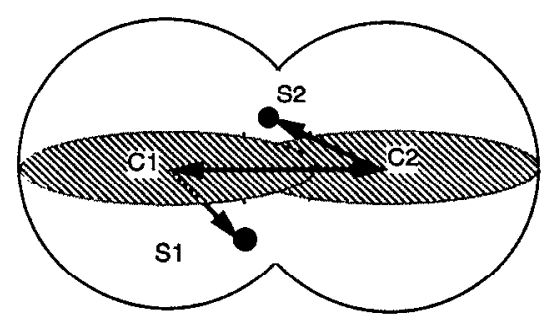

FIG. 17. Dimer formation. 


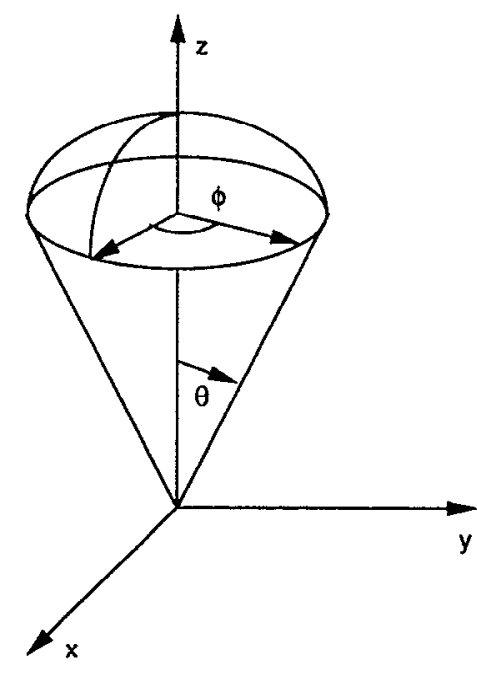

FIG. 18. Calculation of the effective volume and orientation.

namic perturbation terms. We expect the BBMC method presented here to provide a valuable tool for development of such a formalism.

More generally, the BBMC method should be useful for simulation of systems with rapidly varied intermolecular potentials (e.g., ionic fluids). The conventional Metropolis Monte Carlo method "explores" the configurational space of a system in a "blind" and somewhat arbitrary fashion to locate energetically favorable configurations. In many instances, however, these configurations are separated by high energy barriers (such as those arising from Coulombic interactions) which makes them almost inaccessible to a random search. A formalism based on the BBMC method is expected to be more successful because it directs the search to regions of configurational space that are favorable and contribute significantly to the partition function of the system. These regions of space are identified by considering the particular characteristics of a specific system.

\section{ACKNOWLEDGMENTS}

The authors are grateful to the National Science Foundation for financial support. One of us (J.J.dP.) is also grateful to the Camille and Henry Dreyfus foundation for a New Faculty Award. Some of the calculations presented in this work were performed at the Pittsburg Supercomputer Center.

\section{APPENDIX}

\section{Insertion with dimer formation}

As was explained earlier, a major step of the biasedGibbs algorithm involves an insertion of a monomer into a position/orientation that results in the formation of a new dimer. This insertion took place according to the following algorithm (Fig. 17):
(1) Choose at random any available monomer (M1).

(2) Evaluate a new vector (called hereafter $C 1 \rightarrow C 2$ ) by rotating the center-site vector $C 1 \rightarrow S 1$ of the selected monomer M1 by a maximum angle of $\theta_{c}$ using Jansoone's method.

(3) Choose a distance $d \leqslant r_{c}$ at random and position the center $C 2$ of the newly inserted particle (M2) on the axis defined by the vector $C 1 \rightarrow C 2$ and at distance $d$ away from the center of monomer M1.

(4) Evaluate the vector $C 2 \rightarrow C 1$ and rotate it by a maximum of $\theta_{c}$ as in step 2. This is the center-site vector of the newly inserted monomer $(C 2 \rightarrow S 2)$.

\section{Evaluation of the "effective volume/orientation"}

According to Eq. (2) and Eq. (3), we can calculate the $V_{\text {effective }}$ and $\Omega_{\text {effective }}$ of Eq. (36) by carrying the following integrations exactly:

$$
\begin{aligned}
V_{\text {eff }} & =\int_{0}^{r_{c}} \int_{0}^{2 \pi} \int_{0}^{\theta_{c}} r^{2} \sin \theta d \theta d \phi d r, \\
& =2 \pi\left(1-\cos \theta_{c}\right) \frac{r_{c}^{3}}{3}
\end{aligned}
$$

for the volume and

$$
\begin{aligned}
\Omega_{\mathrm{eff}} & =\int_{0}^{2 \pi} \int_{0}^{\theta_{c}} r^{2} \sin \theta d \theta d \phi, \\
& =2 \pi\left(1-\cos \theta_{c}\right)
\end{aligned}
$$

for the orientation of the center to site vector (see Fig. 18).

${ }^{1}$ J. M. Prausnitz, R. N. Lichtenthaler, and E. G. de Azevedo, Molecular Thermodynamics of Fluid-Phase Equilibria (Prentice-Hall, New York, [986).

${ }^{2}$ M. S. Wertheim, J. Stat. Phys. 35, 19 (1984).

${ }^{3}$ M. S. Wertheim, J. Stat. Phys. 35, 35 (1984).

${ }^{4}$ J. Kolafa and I. Nezbeda, Mol. Phys. 72, 161 (1991).

${ }^{5}$ M. S. Wertheim, J. Chem. Phys. 88, 1145 (1988).

${ }^{6}$ W. G. Chapman, J. Chem. Phys. 93, 4299 (1991).

${ }^{7}$ J. K. Johnson and K. E. Gubbins, Mol. Phys. 77, 1033 (1992).

${ }^{8}$ J. P. Hansen and I. R. McDonald, Theory of Simple Fluids, 2nd ed. (Academic, New York, 1986).

${ }^{9}$ J. K. Johnson, J. A. Zollweg, and K. E. Gubbins, Mol. Phys. 78, 591 (1992).

${ }^{10}$ A. Z. Panagiotopoulos, N. Quirke, M. Stapleton, and D. J. Tildesley, Mol. Phys. 3, 527 (1988).

${ }^{11}$ M. H. Kalos and P. A. Whitlock, Monte Carlo Methods, Vol. I (Wiley, Ncw York, 1986).

${ }^{12}$ N. Metropolis, A. W. Rosenbluth, M. N. Rosenbluth, A. H. Teller, and E. Teller, J. Chem. Phys. 21, 1087 (1953)

${ }^{13}$ D. Nicholson and N. G. Parsonage, Computer Simulation and the Statistical Mechanics of Adsorption (Academic, New York, 1982).

${ }^{14}$ D. A. McQuarrie, Statistical Mechanics (HarperCollins, New York, 1973). ${ }^{15}$ M. P. Allen and D. J. Tildesley, Computer Simulation of Liquids (Clarendo, London, 1987).

${ }^{16}$ N. B. Vargaftik, Handbook of Physical Properties of Liquids and Gasses; Pure Substances and Mixtures, 2nd ed. (Hemisphere, 1983). 